\title{
Implementation of adaptive neuro fuzzy inference system and back propagation neural network for the appraisal of power system contingency analysis
}

\author{
Yusufu Yakubu 1, ${ }^{*}$, MB Jibril 2, Bara'u Gafai Najashi ${ }^{3}$, Mohammed Mukhtar Magaji ${ }^{4}$ and Usman Aliyu Magaji ${ }^{5}$ \\ ${ }^{1}$ Department of Electrical and Electronic Engineering, University of Abuja, Nigeria. \\ 2 Department of Electrical and Electronic Engineering, Federal polytechnics, Daura. \\ ${ }^{3}$ Department of Electrical and Electronic Engineering, Baze University Abuja, Nigeria. \\ ${ }^{4}$ Management Information System Unit, Yusuf Maitama Sule University, Kano-Nigeria. \\ ${ }^{5}$ Department of Electrical Engineering, Kano University of Science and Technology Wudil, Kano-Nigeria.
}

GSC Advanced Engineering and Technology, 2022, 03(01), 016-028

Publication history: Received on 01 January 2022; revised on 15 February 2022; accepted on 17 February 2022

Article DOI: https://doi.org/10.30574/gscaet.2022.3.1.0024

\begin{abstract}
Power System Security and Contingency analysis is one of the most important tasks in power systems. In operation, contingency analysis assists engineers to operate the power system at a secure and safe operating point where equipment are loaded within their safe operating area (SOA). Power is dispatched to customers with acceptable quality standards. The results of off-line load flow calculations are used to estimate performance indices (PI flow, PI V). MATLAB toolbox was the proposed methodology used for the implementation. The proposed approach for contingency analysis was found to be appropriate for screening and ranking fast voltage and line flow contingencies.
\end{abstract}

Keywords: Contingency analysis; Evaluation; Screening; Ranking; Adaptive neuro-fuzzy interference system (ANFIS); Performance Index; Voltage and flow ranking

\section{Introduction}

Power system security is a method of achieving, planned to maintain the system during the cost of processor activities when the components stop or fail to respond [1]. Transmission and sub-transmission power systems supply many customers and therefore, there is a need for defensive operation for more reliability in the transmission and subtransmission line in case of component failure or malfunctioning [2]. This can be remedy by applying the single contingency policy (SCP) [3]. The system is designed in such a way that if a transmission line or transformer fails to operate, the system as a whole is still able to operate [4].

Back propagation-neural network (BPNN) is a multi-layer feed-forward network trained according to error Back propagation and is one of the networks used to a great extent, and the network can be used to study and store a great deal of mapping relationship of input/output model, and no need to expose in advance the mathematical equation that describes these mapping relations [5]. Its learning rules are to adopt the highest descent method in which the Back propagations used to control the weight value and threshold value of the network to obtain the minimum error sum of square[6]. This paper focuses on the analysis of the characteristics and mathematical theory of the Back Propagation neural network (BPNN) and also points out the fault of the Back Propagation neural network (BPNN) algorithm as well as several methods for improvement [6].

\footnotetext{
${ }^{*}$ Corresponding author: Yusufu Yakubu

Department of Electrical and Electronic Engineering University of Abuja, Nigeria.
} 
Contingency analysis should be used by planning the working condition of the system in other to measure the efficiency of the power system and the requirement for the future expansion of the transmission lines due to load growth, power loss, or increase in generation [3].

Contingency assessment and power system safety are the utmost important tasks by engineers in the operation and planning of bulk power systems[3]. Contingency analysis is used in the planning power system to check the nature by which the ability of the power systems and when the requirement for further expansion of transmission line due to the high increase in load demand or the need for power expansion of production[2] In the operation of power, engineers were helped by running this analysis to control the energy system at a safe operational range at which components are fixed to their protected cut-off limits[7]Power is passed to consumers with efficiency and standard with reliability[8]. The aim and objective of this analysis are to discover the voltage violation or the overload growth within the range of the equipment, and the appropriate measures necessary to overcome these infringements[9]. Contingency identification and the determination of appropriate corrective measure frequently include calculation of full flow demand [10] This analysis is utmost important and play a significant part of evaluating the safety and security of the power system[11]. An electrical power system is said to be in a safe condition when the system operation is maintained within its operation area or suitable ranges[12] taking into consideration that there will be conceivable outcomes of changes in the system (contingencies) and its surroundings[13] Evaluation in power system there is needed for safety and to have a system that will be sufficiently safe, secured, continuous and reliable[14] even when the contingencies are within the realistic case [2] It is a significant task for operational engineers to predict some future events of these instability/contingencies (outages) and implement preventive control activities as efficiently as possible to maintain system continuity, reliability, and stability for the power supply[15].

The aim of this work is to be achieved through the following set of objectives:

- To implement the Newton Rap son Tradition Method and adaptive neorofuzy inference system (ANFIS).

- To identify the contingency and ranking them by their value by running the prediction value using ANFIS.

- To compare the performance of the methods used.

The driving force for this study is to provide useful power system contingency analysis to model for monitoring the effect of contingency on power system grid. This is also important to sustained operation and management of such systems.

\section{Statement of Problem}

The electrical power system is said to be in a safe condition when the system operation is maintained within its suitable ranges, taking into consideration that there will be a conceivable outcome of changes in the system (contingencies) and its surroundings [10]. There are necessary measures for power system safety to maintain a system that will be protected, reliable, and safe that can be continuously be running even under the case of contingency that is credible[16]. With that, they are not continuously appropriate for online usage. Additionally, a lot of IP-based analytical methods go through the difficulty of false alarm and/or misclassification[17]. An effective contingency can be categorized as a noncritical contingency, which is referred to as Miss-classification[9]. When inactive contingency is categorized as critical, then a false alarm has happened. A system that is speedy and fast with the ability to avoid fake alarms must be needed [18]. Nowadays, continuous delivery of electrical energy is of utmost importance due to the societal reliability on the sector in the power system [19].

System security is the fundamental of power survival and since contingency analysis adds security, reliability, and customer services as well as protecting the power system from hazard or harm [3]. Some of the methods lack quality due to some set back that is the rule-based system and system-specific even though they are fast[18]. With recent advances in soft computing learning techniques, Adaptive Neuro fuzzy Inference system (ANFIS), technique for contingency screening and ranking will be a good option [5]. Furthermore, by using Adaptive Neuro fuzzy Inference system (ANFIS), [20].

\section{Material and methods}

\subsection{Adaptive neuro-fuzzy interference system (ANFIS)}

The neuro-fuzzy network is a five-layer feed-forward network that maps an input space to an output space using neural network learning algorithms and fuzzy reasoning. Figure.0 depicts the ANFIS architecture, and the following is a description of the model. 


\subsubsection{Layer 1}

In this layer, each node adapts to a function parameter. The result from each node is a degree of membership value, which is determined by the membership functions' input [21]

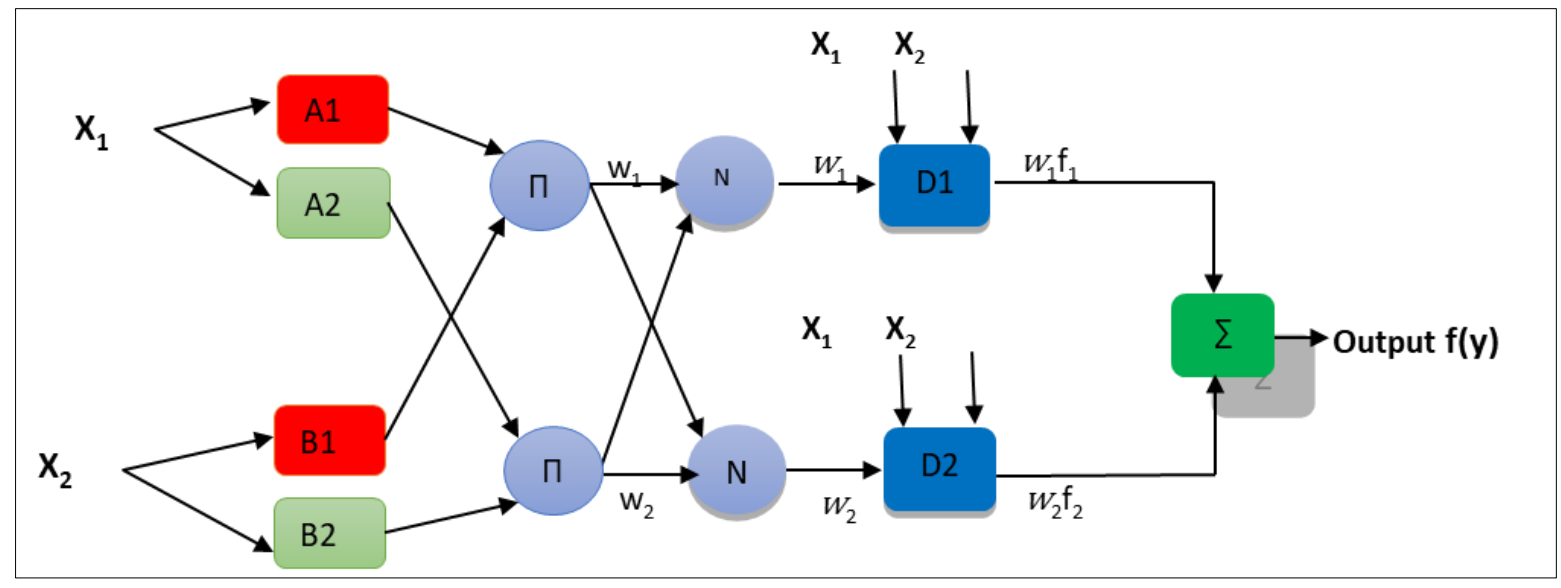

Figure 1 ANFIS architecture

$$
\begin{gathered}
\mu_{A i}(x)=\exp \left[-\left(\frac{x-c_{i}}{2 a_{i}}\right)^{2}\right] \ldots \\
\mu_{A i}(x)=\frac{1}{1+\left|\frac{x-c_{i}}{a_{i}}\right|^{2 b} \ldots \ldots} \\
O_{1, i}=\mu_{A i}(x), i=1,2 \ldots \ldots \\
O_{1, i}=\mu_{B i-2}(y), i=3,4 \ldots \ldots
\end{gathered}
$$

where $\mu_{A i}$ and $\mu_{B i-2}$ are the degree of membership functions for the fuzzy sets $A_{i}$ and $B_{i}$ respectively, and $\left\{a_{i}, b_{i}, c_{i}\right\}$ are the parameters of a membership function that can change the shape of the membership function. Premise parameters are the terms used to describe the parameters in this layer [22].

\subsubsection{Layer 2}

Every node in this layer is fixed or non-adaptive, and the circle node is labelled as $\prod$. The output node is the result of multiplying of signal coming into the node and delivered to the next node. Each node in this layer represents the firing strength for each rule. In the second layer, the T-norm operator with general performance, such as the AND, is applied to obtain the output;

$$
O_{2 i}=w_{i}=\mu_{A i}(x) * \mu_{B i}(y), i=1,2
$$

Where $w_{i}$ is the output that represents the firing strength of each rule.

\subsubsection{Layer 3}

This layer's nodes are either fixed or non-adaptive, with the circle node labelled as N. The ratio between the $i$-th rule's firing strength and the sum of all rules' firing strengths is calculated at each node. The normalized firing strength is the name given to this result [23]

$$
O_{3 i}=\overline{w_{l}}=\frac{w_{i}}{\sum_{i} w_{i}} \ldots
$$

\subsubsection{Layer 4}

In this layer, every node is an adaptive node to output, with a node function defined as

$$
O_{4 i}=\bar{w}_{i} f_{i}=\bar{w}_{i}\left(p_{i} x+q_{i} y+r_{i}\right)
$$


where $\bar{w}_{i}$ is the normalized firing strength from the previous layer (third layer) and $\left(p_{i} x+q_{i} y+r_{i}\right)$ is a parameter in the node. Consequent parameters refer to the parameters in this layer.

\subsubsection{Layer 5}

This layer's single node is a fixed or non-adaptive node that sums all incoming signals from the previous node to compute the overall output. A circle node is labeled as $\sum$ in this layer.

$$
O_{5 i}=\sum_{i} \bar{w}_{i} f_{i}=\frac{\sum_{i} \bar{w}_{i} f_{i}}{\sum_{i} \bar{w}_{i}} \ldots \ldots
$$

Learning rules are used to govern the neuron weights updating process and the procedure of utilizing the learning rules to update the weights is known as learning algorithm. Based on the learning procedure, neural networks are categorized as supervised or unsupervised or hybrid.

In supervised learning, the neural network is provided with inputs and the desired outputs. The main concern is to obtain a set of weights that drastically reduces the error between the network output and the desired output. Unsupervised learning uses only input, the network updates its weights so that similar input yields corresponding output. Hybrid learning combines supervised and unsupervised learning [24].

Neural network gains vast popularity over the last few decades, particularly in the field of system identification, modeling and control applications. The most common applications are future extraction, pattern recognition, classification and prediction [16].

In conducting the research, the contingency selection technique will be grounded on the performance index (PI) which might signify either a line overload or a bus voltage drop limit violation, the Performance Index will be calculated using traditional Newton Raphson, Adaptive Neurofuzzy inference System (ANFIS) [24]. A huge number of patterns will be generated at random for an individual bus within a wide range of load differences. In each pattern, a full AC load flow will be carried out to calculate the line flows before failure and the voltages at the terminals of the line or the possibly the generator, and so also the equivalent to the unavailability of line and generator to calculate the voltage indices and flow performance. The recommended technique will be planned and tested using Adaptive neurofuzzy inference system (ANFIS) on a Windows environment using MATLAB toolbox. The accuracy of the recommended method will be illustrated by contingency screening and ranking in the 6-bus system. The functioning of the recommended technique will be compared with the traditional Newton Raphson method.

\subsection{Performance Evaluation}

The accuracy of forecasting models is the most important element in determining their performance success [25]-[29]. As a result, the generally used error metrics are used to evaluate the outputs of prediction models as well as to compare them to one another. Metrics such as Coefficient of determination ( $\left.\mathrm{R}^{2}\right)$, Correlation coefficient (R), Mean square error (MSE) and Root mean square error (RMSE) were used to compare the performance success of the forecasting models used in this study more information on performance evaluation can be found in the following references [30],[31], [32], $[33],[34],[35],[36],[37],[38],[39],[23],[40],[41],[42],[43],[44],[45],[46]$ and [47].

$$
\begin{gathered}
R^{2}=1-\frac{\sum\left(x_{i}-y_{i}\right)^{2}}{\sum\left(x_{i}-\bar{x}_{i}\right)^{2}} \ldots \ldots(1) \\
R=\sqrt{\left(1-\frac{\sum\left(x_{i}-y_{i}\right)^{2}}{\sum\left(x_{i}-\bar{x}_{i}\right)^{2}}\right) \ldots \ldots(2)} \\
M S E=\frac{1}{n} \sum_{i=1}^{n}\left(x_{i}-y_{i}\right)^{2} \ldots \ldots(3) \\
\left.R M S E=\sqrt{\left(\frac{1}{n} \sum_{i=1}^{n}\left(x_{i}-y_{i}\right)^{2}\right) \ldots \ldots .(}\right)
\end{gathered}
$$

where $x_{i}$ are values of the $\mathrm{x}$-variable in a sample, $y_{i}$ are values of the $\mathrm{y}$-variable in a sample, $\bar{x}_{i}$ is the mean of the values of the $\mathrm{x}$-variable and $n$ is the number of data points. 


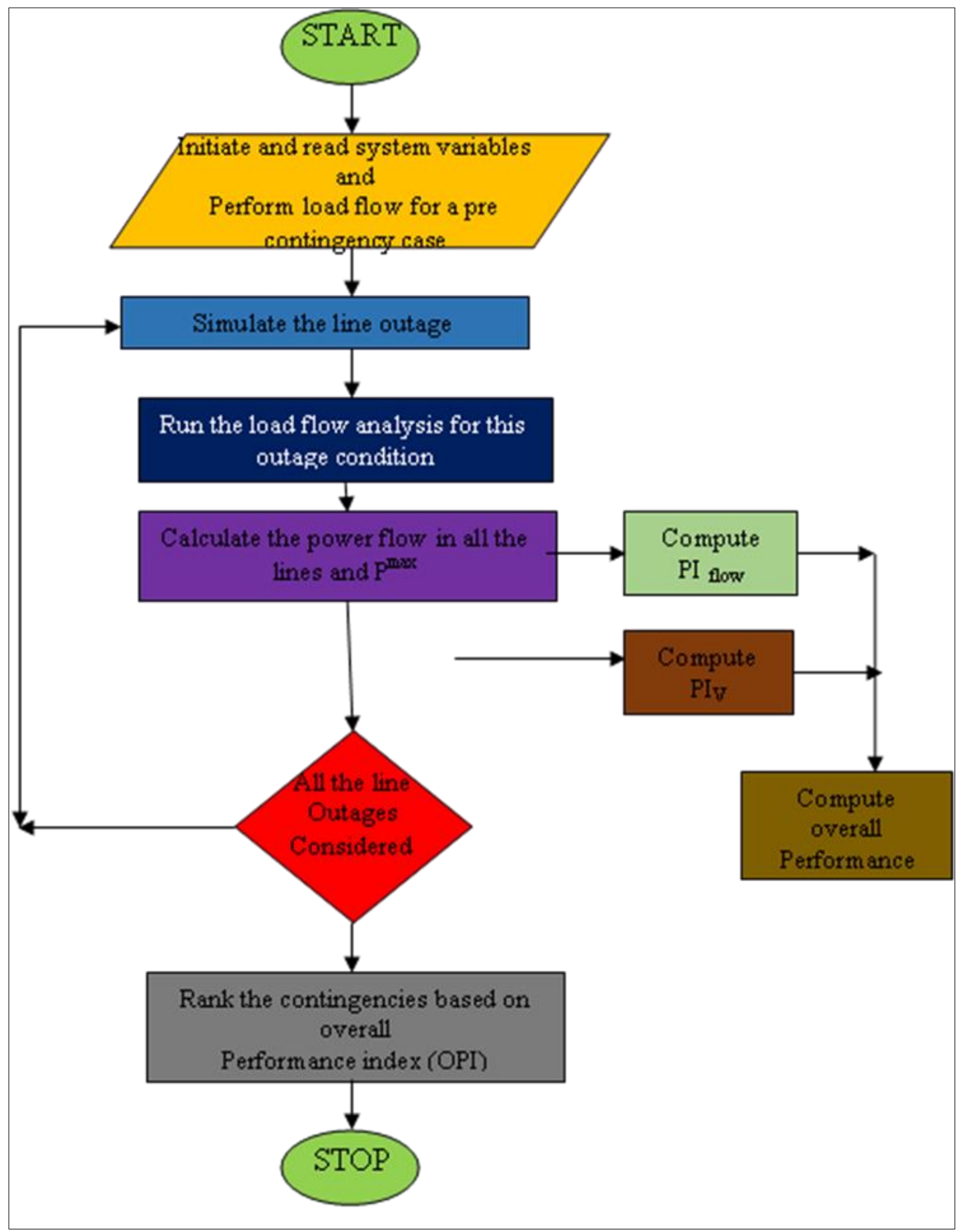

Figure 2 Flow chart Algorithm 


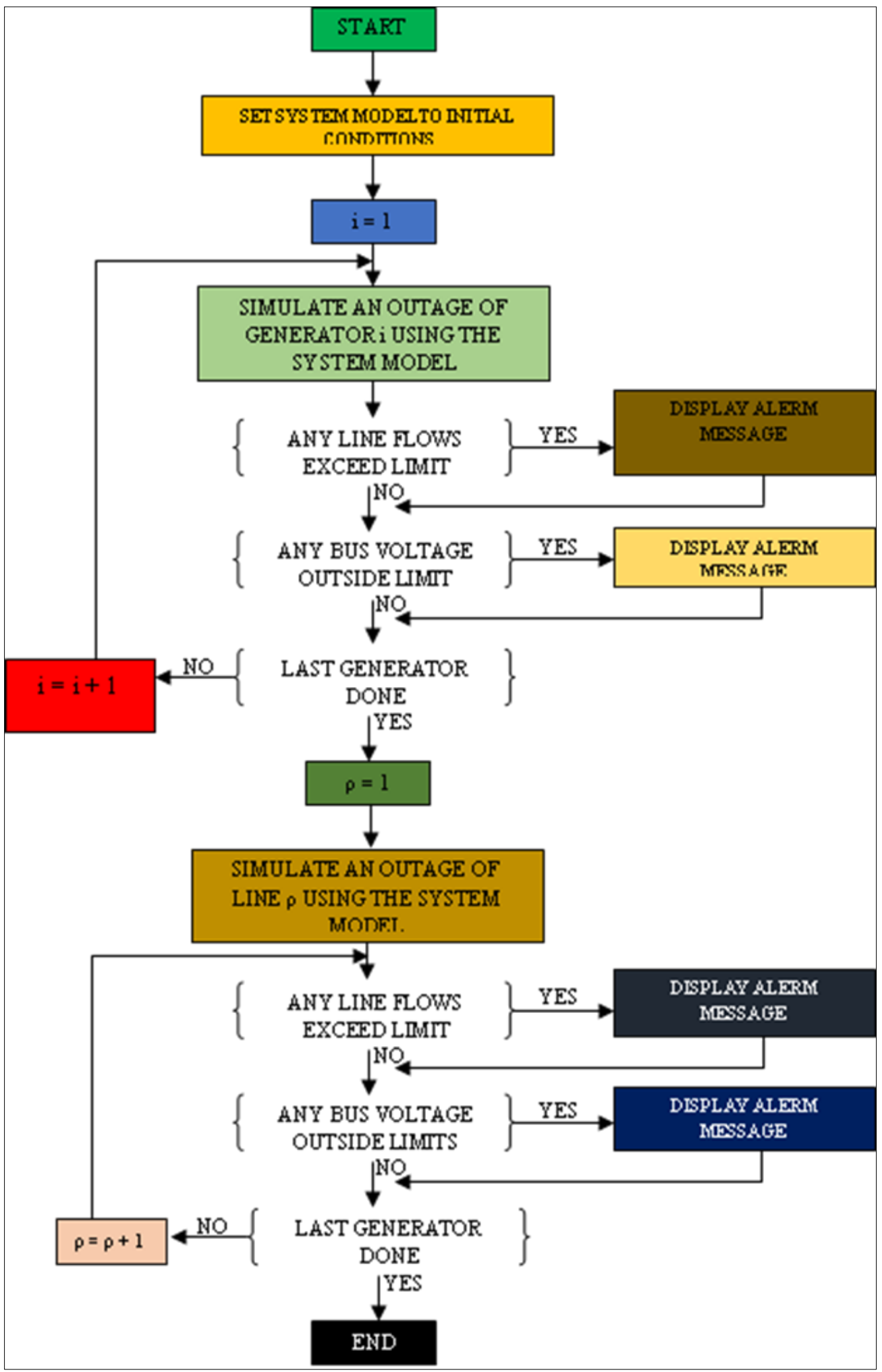

Figure 3 Full AC power flow contingency analysis procedure 


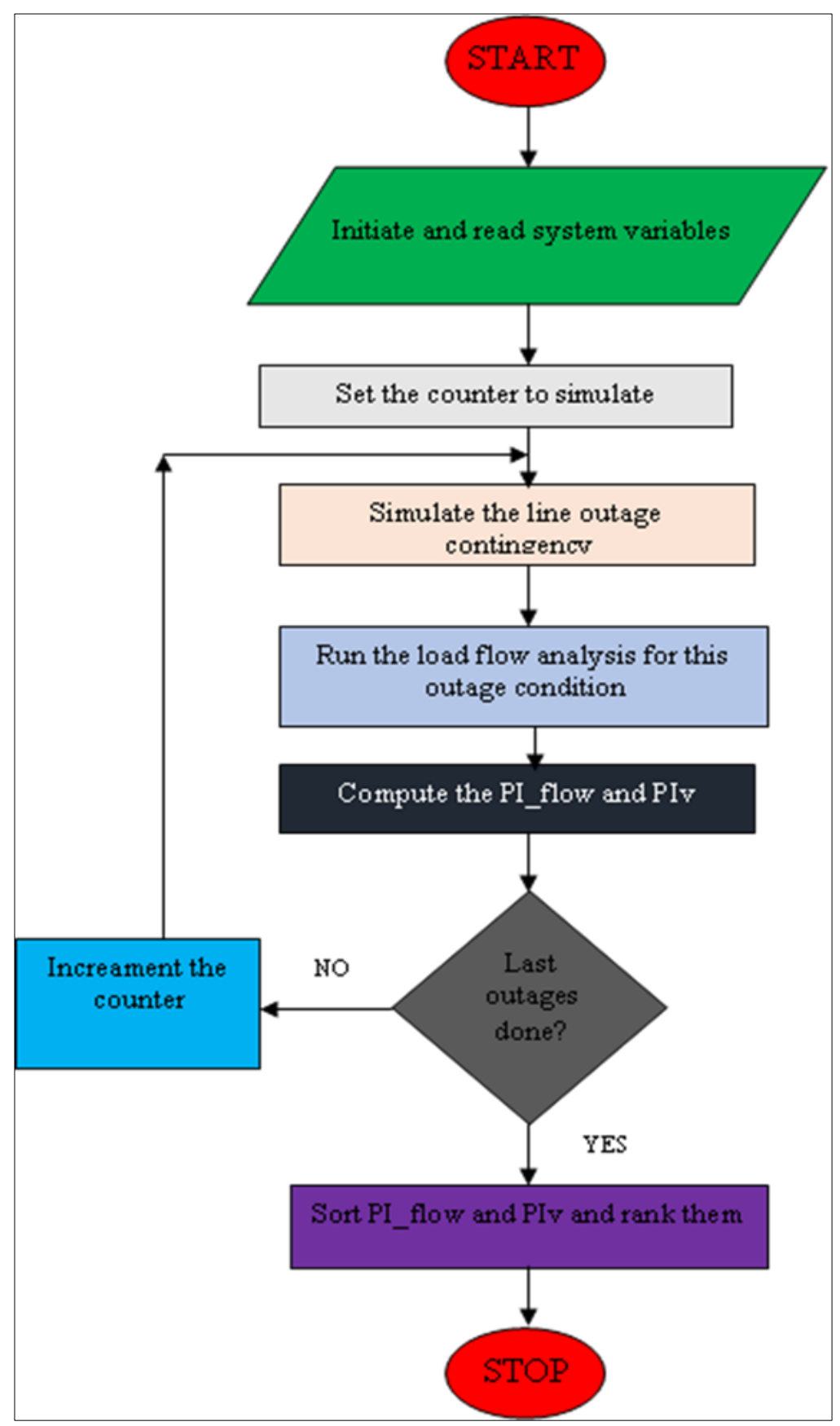

Figure 4 A flow chart for line outage contingency selection technique

\section{Results and discussion}

This study presented the comparative analysis of both BPNN nonlinear artificial intelligence model (ANFIS) for the estimation ofImplementation of adaptive Neuro Fuzzy inference system, and Back Propagation Neural Network for the Appraisal of Power system Contingency analysisthe modeling results were evaluated using $\mathrm{R}^{2}$, R, RMSE, and MSE in both training and testing phase.

The most dominant and suitable input combinations with the targeted variables were investigated using traditional sensitivity analysis and a correlation matrix. The type of linear relationship between the variables is represented in Table. It can also be used as a basic indicator for the correlation of variable sets as shown 2 that stationary and significant variables with probability less than $0.05(\mathrm{P}<0.05)$ indicates the high strength of the linear correlations. Also, the negative correlation values show an inverse relationship between two variables. As a result, the correlation value's weakness 
indicates that traditional methods are ineffective in modeling such complex interactions and that there is a significant need to introduce more robust tools.

Table 1 Correlation between the experimental variables

\begin{tabular}{|l|l|l|l|l|}
\hline Parameters & Load 1 & Load 2 & Load 3 & Observed load \\
\hline Load 1 & 1 & & & \\
\hline Load 2 & 0.363272 & 1 & & \\
\hline Load 3 & 0.054223 & 0.679186 & 1 & \\
\hline Observed load & 0.697553 & 0.255876 & 0.231663 & 1 \\
\hline
\end{tabular}

From the results in Error! Reference source not found. the model combinations were generated, from M1, M2, using ANFIS. Error! Reference source not found. shows the variables that were used as inputs for each model, load $1+$ load 2 where used as input to forecast observed load with Adaptive Neuro Fuzzy inference system (ANFIS) Designer tool of MATLAB was used. The input and output parameters of the membership function (MF) were tuned so as to generate a Sugeno-type fuzzy inference system. For the input parameter a triangular MF type was selected and for the output parameter, a constant MF type was selected. The FIS was trained with an error tolerance of 0.005 for 50 iterations (epochs).

Table 2 Models and corresponding variables

\begin{tabular}{|c|l|}
\hline Models (M) & Variables \\
\hline M1 & Load $1+\operatorname{load} 2$ \\
\hline M2 & Load $1+\operatorname{Load} 2+$ Load 3 \\
\hline
\end{tabular}

Table 3 Predicted results of ANFIS based on the performance evaluation criteria

\begin{tabular}{|l|l|l|l|l|l|l|l|l|}
\hline & \multicolumn{4}{|l|}{ TRAINING PHASE (75\%) } & \multicolumn{4}{l|}{ TESTING PHASE (25\%) } \\
\hline & $\mathbf{R}^{\mathbf{2}}$ & $\mathbf{R}$ & MSE & RMSE & $\mathbf{R}^{\mathbf{2}}$ & $\mathbf{R}$ & MSE & RMSE \\
\hline ANFIS-M1 & 0.7640 & 0.8740 & 0.0135 & 0.1162 & 0.7690 & 0.8769 & 0.0312 & 0.1765 \\
\hline ANFIS-M2 & 0.9620 & 0.9808 & 0.0022 & 0.0466 & 0.3998 & 0.6323 & 0.0082 & 0.0904 \\
\hline
\end{tabular}

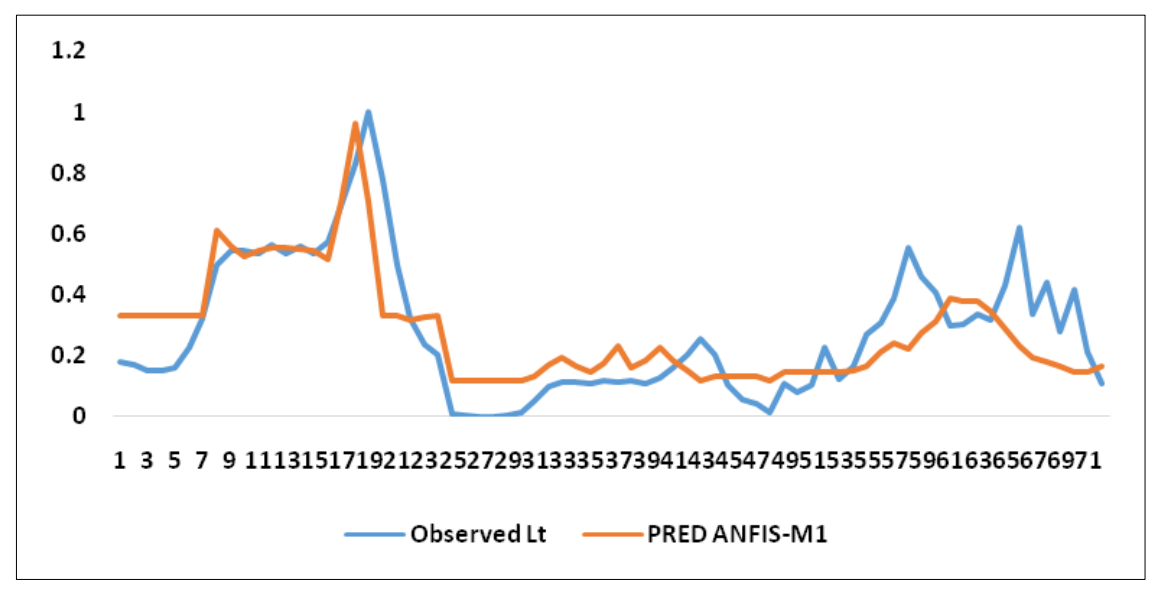


Figure 5 Time Series Plot ANFIS -M1

The forecasted observed load produced by the ANFIS model were partitioned into training (75\%) and testing (25\%) in order to properly evaluate the performance of ANFIS in forecasting observed load. The results of the performance criteria are displayed in Table. It can be seen that the ANFIS models, ANFIS-M2 produced the best results with values of $\mathrm{R}^{2}=0.9620, \mathrm{R}=0.9808, \mathrm{MSE}=0.0022$, and $\mathrm{RMSE}=0.0466$. ANFIS model is more accurate.

For further understanding, the results produced by the best models from ANFIS Model 1 and Model 2 are analyzed using a time series plot to show the degree of agreement between the observed Load demand and the predicted load demand values. When such variables in the plot overlap this implies that there is a high degree of agreement between the variables being plotted as shown in figure 5 and figure 6 of series plots ANFIS Model 1 and ANFIS Model 2

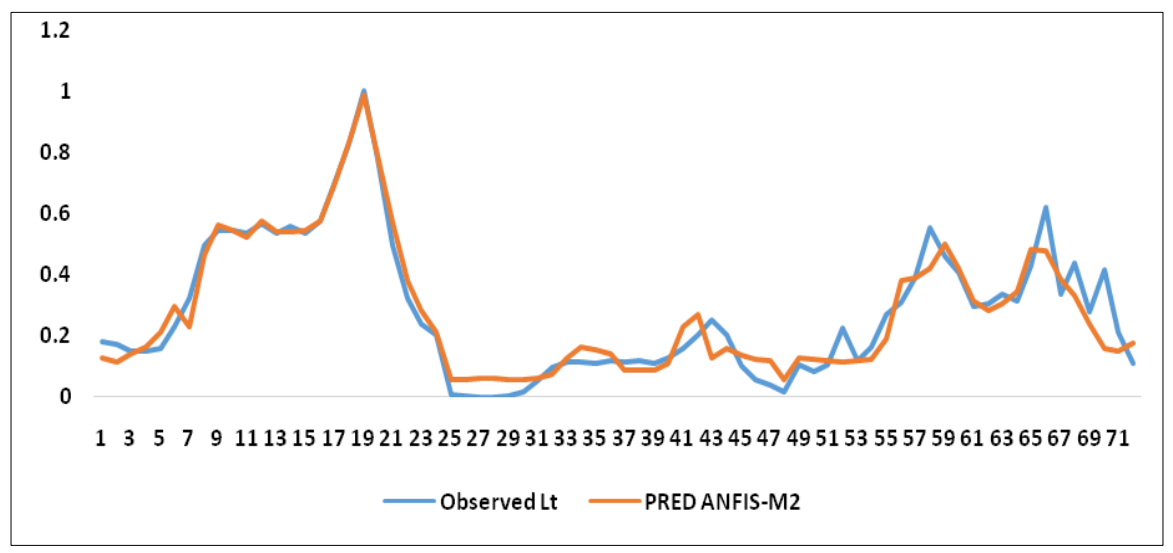

Figure 6 Time Series Plot ANFIS -M2

From the scatter plots in figure 7 below for ANFIS M1 has performance of $\left(\mathrm{R}^{2}\right)$ with $49 \%$ accuracy between the observed and predicted values.

While fig 3.7 below, it can be clearly seen that ANFIS M2 model has the highest performance criteria( $\left.\mathrm{R}^{2}\right)$ with $92 \%$ accuracy between the observed and predicted values. The performance of the ANFIS model could be associated with its ability to combine both the knowledge of ANN and fuzzy logic. With regards to performance accuracy, ANFIS model2on average outperformed ANFIS Model 1 respectively in the verification phase.

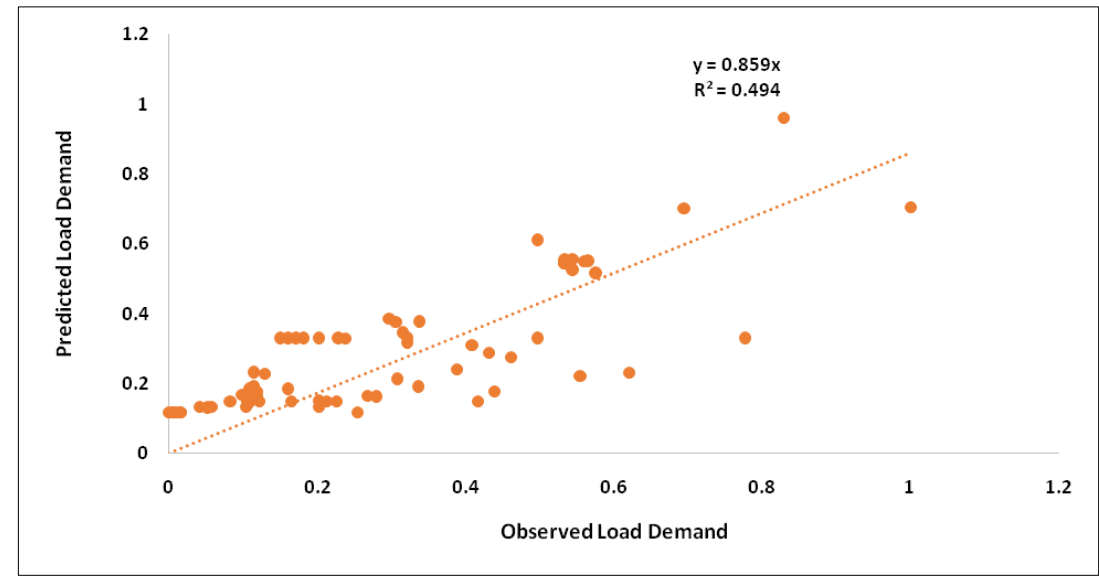

Figure 7 Predicted Load ANFIS-M1

From the error plots of the testing in figure 9 below, the roots mean square error (RMSE) of ANFIS M1 is higher than the roots mean square error (RMSE) of ANFIS M2 likewise the mean square error (MSE) of ANFIS M2 is higher than the mean square error (MSE) of ANFIS M1, that's to shows that the ANFIS M2 gives a better result as compared to ANFIS M1 the higher the roots mean square error, the poor the result, the lower the mean square error, the more accuracy the result. In the case of ANFIS, the roots mean square error (RMSE) of ANFIS M1 is higher than the roots mean square error 
(RMSE) of ANFIS M2, and the mean square error (MSE) of ANFIS M1 is higher than the (MSE) of ANFIS M2, hence ANFIS M2 gives a better result as compared with ANFIS M1.The figure below is the combination of error plot for training and testing ANFIS.

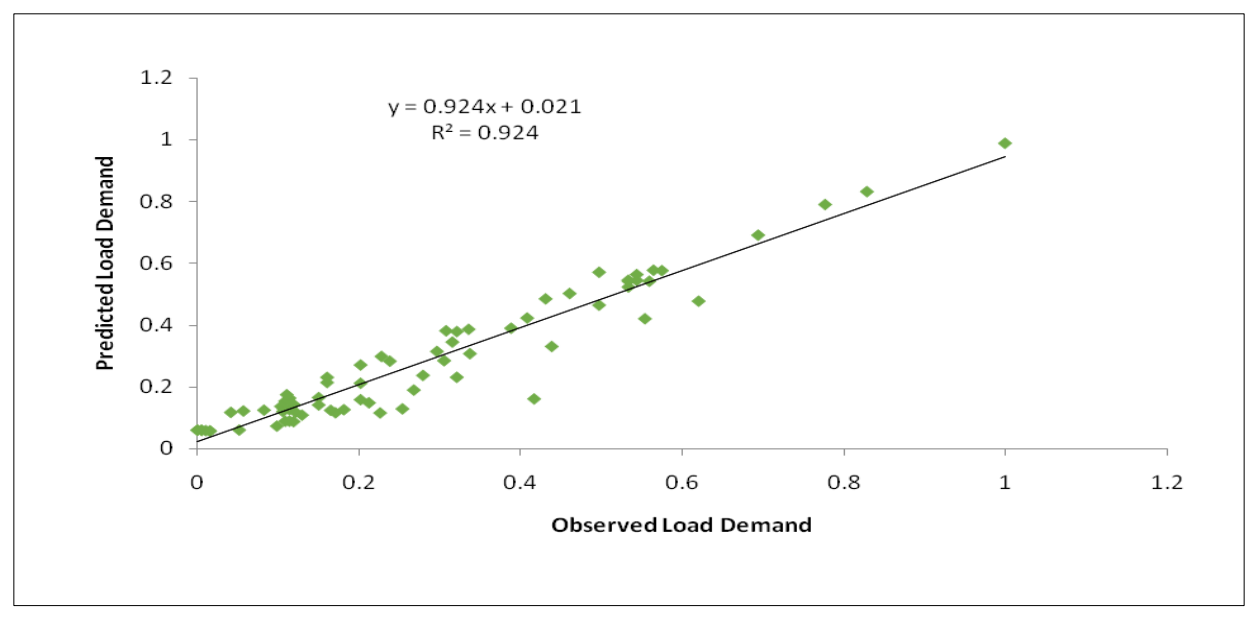

Figure 8 Predicted Load ANFIS-M2

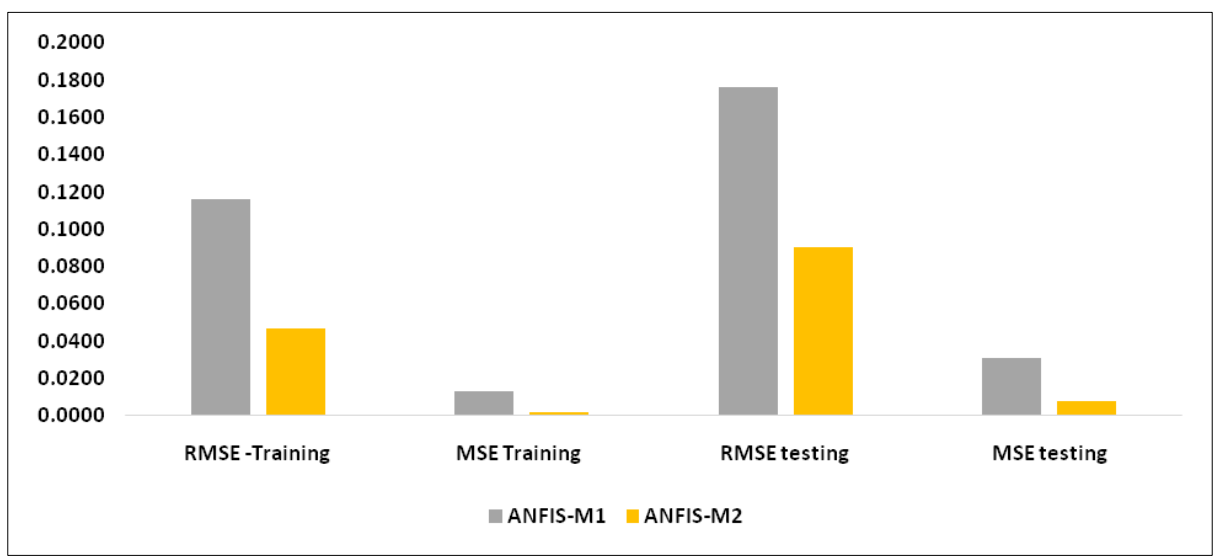

Figure 9 Error plot for Training and Testing of ANFIS

\section{Conclusion}

It can be clearly observed that Adaptive Neuro Fuzzy Inference System (ANFIS) gives out a better result as compared in the columns of tables that correspond to the contingency ranking load condition as it is nearer to the same obtained by the Analytical technique (NR) compared to Artificial Neural Network (ANN).

The voltage and flow performance index values displayed nearest result for both methods i.e. ANFIS and NR, as such those with most severe contingencies have a greater performance index value. Also, both the techniques have the same sequence in their contingency ranking grade except in place of contingencies 1 and 2, which display a close value result of the performance indices, so they have less effect as seen on the system, which can be ignored.

The analytical technique for analyzing contingency have several computations of performance indices, therefore, performing complete AC load flow for the overall contingencies with the approach, is complex and required more time to analyze. With this, ANFIS approach to this technique can be more suitable which consist of screening and training module for different contingencies that tally with the voltage violation in the buses and power flow violation in the lines. Accurate result was obtained from the screening module where performance indices (Pinfold, PIv) for the unknown load variation patterns are obtained when compared with analytical technique. Ranking module was used to rank the 
screened performance indices according to the severity of the contingencies. ANFIS approach demonstrated on the standard IEEE 6-bus system for contingency technique has been tested and gives better results.

According to the simulation results obtained in the above table, it was observed that:

- $\quad$ ANFIS approach to this technique provides speed computation in the process of generating variable load data for voltage and flow performance index respectively.

- Effect of masking is avoided in the contingencies, if the performance indices are properly constructed.

- Once the training and evaluation of the network is done, it provides speed contingency screening and less calculation as compared to other techniques.

- ANFIS approach to contingency analysis for online applications is a good assessment tool appropriated for power system management.

The work done in this research uses Newton Rap son method to generate the data on a standard IEEE 6-Bus system, under different loading conditions which are further utilized to train ANN and ANFIS for further comparison of their performances. In line with the study made, further work can be suggested by using different algorithms and on different standard IEEE bus system.

\section{Compliance with ethical standards}

\section{Acknowledgments}

The authors would like to appreciate the University of Abuja and Base University for Implementation of Adaptive Neuro Fuzzy Inference System, And Back Propagation Neural Network for The Appraisal of Power System Contingency Analysis which provided the data used to carry out this research study. The authors would also like to appreciate Dr. Bara'u Gafai Najashi, Dr. S.I. Abba.

\section{Disclosure of conflict of interest}

The authors declared that there is no any conflict of interest in this research.

\section{References}

[1] M Albadi. Power Flow Analysis. Comput. Model. Eng. 2020; 2: 1-21.

[2] F Sch. Contingency Analysis of Power Systems with Artificial Neural Networks. 2018.

[3] F Schafer, JH Menke, M Braun. Contingency Analysis of Power Systems with Artificial Neural Networks. 2018 IEEE Int. Conf. Commun. Control. Comput. Technol. Smart Grids, SmartGridComm. 2018.

[4] YS, Sunday, OP Ubeh, AA Saidu, M Illiasu, J Boyi. Load-ability Analysis during Contingency with Unified Power Flow Controller Using Grey Wolf Optimization Technique. 2020; 4(2): 23-31.

[5] MJ Cooke, GL Lebby. A Backpropagation Based Alternative to the Classical Power Flow Algorithm.

[6] J Li, J Cheng, J Shi, F Huang. Brief Introduction of Back Propagation (BP ) Neural Description of BP Algorithm in Mathematics. 2012; 2: 553-554.

[7] RLY Chen, A Cohn, N Fan, A Pinar. Contingency-risk informed power system design, IEEE Trans. Power Syst. 2014; 29(5): 2087-2096.

[8] S Robak, J MacHowski, K Gryszpanowicz. Contingency selection for power system stability analysis, Proc. 2017 18th Int. Sci. Conf. Electr. Power Eng. EPE. 2017; 0-4.

[9] AY Dewi, SP Hadi. Contingency Analysis of Power System Electrical Operation. 2007; 875-878.

[10] K Verma et al. Kusum Verma K. R. Niazi. 2012; 1: 1-12.

[11] J Choi, TD Mount, RJ Thomas. Transmission expansion planning using contingency criteria, IEEE Trans. Power Syst. 2014; 22(4): 2249-2261.

[12] WD Oliveira, JPA Vieira, UH Bezerra, DA Martins, B das, G Rodrigues. Power system security assessment for multiple contingencies using multiway decision tree. Electr. Power Syst. Res. 2017; 148: 264-272. 
[13] RD Christie, BF Wollenberg, I Wangensteen. Transmission management in the deregulated environment. Proc. IEEE. 2014; 88(2): 170-195.

[14] Alwafi Ridho Subarkah. No Title Effect of Turmeric Ethanol Extract Ointment (Curcuma Longa) on the Healing of Grade II Burns in Wistar Rats (Rattus Norvegicus. 2018; 151(2): 10-17.

[15] SI Abba, G Elkiran. Effluent prediction of chemical oxygen demand from the astewater treatment plant using artificial neural network application. Procedia Comput. Sci. 2017; 120: 156-163.

[16] K Verma, KR Niazi. Supervised learning approach to online contingency screening and ranking in power systems. Int. J. Electr. Power Energy Syst. 2012; 38(1): 97-104.

[17] AY Abdelaziz, ATM Taha, MA Mostafa, AM Hassan. Fuzzy Logic Based Power System Contingency Ranking, Int. J. Intell. Syst. Appl. 2013; 5(3): 1-12.

[18] LM Adesina, O Ogunbiyi. Newton-Raphson Algorithm for Power Flow Solution and Application Newton-Raphson Algorithm for Power Flow Solution and Application. May 2020.

[19] U Uzubi, E Ejiogu. Artificial Neural Network Technique for Transmission Line Protection on Nigerian Power System. 2017; 52-58.

[20] GA Adepoju, MSc SO, A Ogunjuyigbe MSc, KO Alawode, B Tech. Application of Neural Network to Load Forecasting in Nigerian Electrical Power System Application of Neural Network to Load Forecasting in Nigerian Electrical Power System. May 2014, 2007.

[21] AG Usman, MH Ahmad, N Danraka, SI Abba. The effect of ethanolic leaves extract of Hymenodictyon floribundun on inflammatory biomarkers : a data - driven approach. Bull. Natl. Res. Cent. 2021.

[22] SI Abba. Modeling of Water Treatment Plant Performance using Artificial Neural Network: Case Study Tamburawa Kano-Nigeria, Dutse J. Pure Appl. Sci. 2020; 6(3): 135-144.

[23] MH Ahmad, AG Usman, SI Abba. Comparative performance of extreme learning machine and HammersteinWeiner models for modelling the intestinal hyper-motility and secretory inhibitory effects of methanolic leaf extract of Combretumhypopilinum Diels (Combretaceae), Silico Pharmacol. 2021; 9(1).

[24] AG Usman, S Işik, SI Abba, F Meriçli. Chemometrics-based models hyphenated with ensemble machine learning for retention time simulation of isoquercitrin in Coriander sativum L. using high-performance liquid chromatography, J. Sep. Sci. 2021; 44(4): 843-849.

[25] SI Abba, SJ Hadi, J Abdullahi. River water modelling prediction using multi-linear regression, artificial neural network, and adaptive neuro-fuzzy inference system techniques. Procedia Comput. Sci. 2017; 120: 75-82.

[26] UM Ghali, M Alhosen, A Degm, AN Alsharksi, Q Hoti, AG Usman. Development Of Computational Intelligence Algorithms For Modelling The Performance Of Humanin And Its Derivatives In HPLC Optimization Method Development. 2020; 9(8): 110-117.

[27] AG Usman, S Işik, SI Abba. A Novel Multi-model Data-Driven Ensemble Technique for the Prediction of Retention Factor in HPLC Method Development, Chromatographia. 2020.

[28] HU Abdullahi, AG Usman, SI Abba. Modelling the Absorbance of a Bioactive Compound in HPLC Method using Artificial Neural Network and Multilinear Regression Methods. 2020; 6(2): 362-371.

[29] AG USMAN, S IŞIK, SI ABBA, F MERİÇLİ. Artificial intelligence-based models for the qualitative and quantitative prediction of a phytochemical compound using HPLC method, Turkish J. Chem. 2020; 44(5): 1339-1351.

[30] GM Khalid, AG. Usman. Application of data-intelligence algorithms for modeling the compaction performance of new pharmaceutical excipients. 2021.

[31] SS. Sammen et al., Assessment of climate change impact on probable maximum floods in a tropical catchment, Theor. Appl. Climatol. 2022; 0123456789.

[32] AG Usman, S Işik, SI Abba, F Meriçli. Chemometrics-based models hyphenated with ensemble machine learning for retention time simulation of isoquercitrin in Coriander sativum L. using high-performance liquid chromatography, J. Sep. Sci. December 2020; 1-7.

[33] H Tao et al., Development of new computational machine learning models for longitudinal dispersion coefficient determination: case study of natural streams, United States, Environ. Sci. Pollut. Res., Ml. 2022. 
[34] UM Ghali et al., Applications of Artificial Intelligence-Based Models and Multi- Linear Regression for the Prediction of Thyroid Stimulating Hormone Level in the Human Body. 2020; 29(4): 3690-3699.

[35] W Asnake Metekia, A Garba Usman, B Hatice Ulusoy, S Isah Abba, K Chirkena Bali. Artificial intelligence-based approaches for modeling the effects of spirulina growth mediums on total phenolic compounds, Saudi J. Biol. Sci., xxxx. 2021.

[36] SI Abba et al. Comparative implementation between neuro-emotional genetic algorithm and novel ensemble computing techniques for modelling dissolved oxygen concentration. Hydrol. Sci. J. 2021.

[37] UM Ghali et al., Advanced chromatographic technique for performance simulation of anti-Alzheimer agent: an ensemble machine learning approach. SN Appl. Sci. 2020; 2(11).

[38] SI Abba, AG Usman. Modeling of Water Treatment Plant Performance using Artificial Neural Network: Case Study Tamburawa Kano-Nigeria. September 2020.

[39] UM Ghali et al., Advanced chromatographic technique for performance simulation of anti-Alzheimer agent: an ensemble machine learning approach, SN Appl. Sci. 2020; 2(11).

[40] AG Usman, S Işik, SI Abba. Hybrid data-intelligence algorithms for the simulation of thymoquinone in HPLC method development, J. Iran. Chem. Soc. 2021; 18(7): 1537-1549.

[41] AG Usman, S Isik, SI Abba, F Mericli. Artificial intelligence based models for the qualitative and quantitative prediction of a phytochemical compound using HPLC method, Turkish J. Chem. 2021; 44(5).

[42] UM Ghali et al., Applications of Artificial Intelligence-Based Models and Multi-Linear Regression for the Prediction of Thyroid Stimulating Hormone Level in the Human Body, Int. J. Adv. Sci. Technol. 2020; 29(4): 36903699.

[43] AG Usman, MH Ahmad, RN Danraka, SI Abba. The effect of ethanolic leaves extract of Hymenodictyon floribundun on inflammatory biomarkers: a data-driven approach, Bull. Natl. Res. Cent. 2021; 45(1).

[44] SI Haruna et al., Compressive Strength of Self-Compacting Concrete Modified with Rice Husk Ash and Calcium Carbide Waste Modeling: A Feasibility of Emerging Emotional Intelligent Model (EANN) Versus Traditional FFNN, Arab. J. Sci. Eng. 2021; 46(11): 11207-11222.

[45] SI Malami et al., Implementation of soft-computing models for prediction of flexural strength of pervious concrete hybridized with rice husk ash and calcium carbide waste, Model. Earth Syst. Environ. 2021.

[46] K Mahmoud et al., Prediction of the effects of environmental factors towards COVID-19 outbreak using AI-based models, IAES Int. J. Artif. Intell. 2021; 10(1): 35-42.

[47] SI Abba et al. Comparative implementation between neuro-emotional genetic algorithm and novel ensemble computing techniques for modelling dissolved oxygen concentration, Hydrol. Sci. J. 2021; 66(10): 1584-1596. 